\section{Atemnot als Spättypreaktion auf Colistin}

Colistin, ein Antibiotikum aus der Gruppe der Polymyxine, wird vor allem gegen multiresistente gramnegative Erreger bei Risikogruppen wie beispielsweise Patienten mit Cystischer Fibrose eingesetzt. Aufgrund der hohen Neuro- und Nephrotoxizität bei systemischer Gabe wird das Medikament als Inhalationstherapie verabreicht. Bei etwa jedem zehnten Patienten kommt es dabei zu pulmonalen Beschwerden wie Husten, Brustenge und Bronchokonstriktion. Als Ursachen werden verschiedene Pathomechanismen wie eine Irritation der Bronchien durch den Wirkstoff oder durch Zusatzstoffe diskutiert.

Dass auch eine T-Zell-vermittelte allergische Spättypreaktion zu Atemnot führen kann, zeigt ein Fall aus dem Allergiezentrum der LMU München, den Dr. Rita Varga vorstellte. Der Patient, der an Cystischer Fibrose litt, hatte innerhalb von fünf Jahren dreimal eine Colistin-Inhalationstherapie erhalten. Jedes Mal kam es zu schwerer Atemnot, die intensivmedizinisch behandelt werden musste. Der Abstand zwischen Beginn der Therapie und Auftreten der Atembeschwerden ver- ringerte sich von zunächst sechs Wochen auf acht und schließlich auf nur zwei Tage. Colistin wurde jedes Mal sofort abgesetzt.

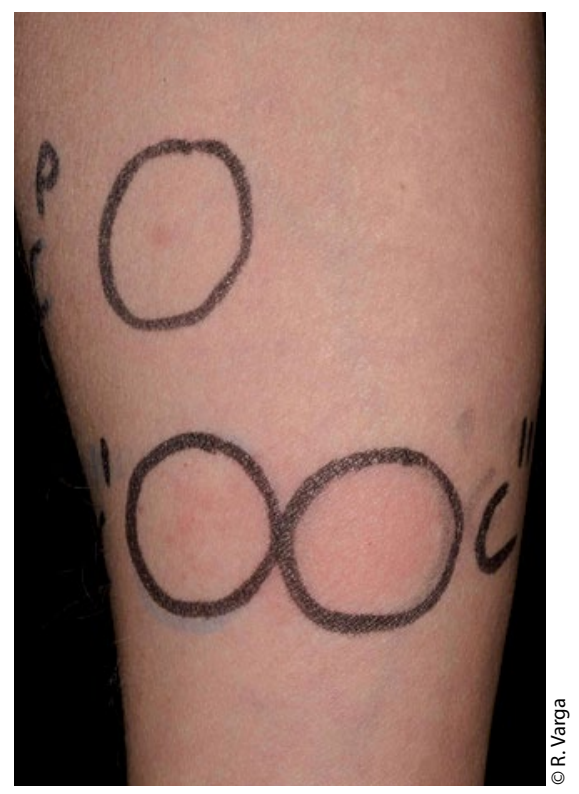

Spätablesung der Pricktestung mit Colistin
Der Patient stellte sich zehn Jahre nach der letzten Reaktion vor, um die Möglichkeit einer erneuten Anwendung überprüfen zu lassen.

Im Pricktest trat bei einer Konzentration von $80 \mathrm{mg} / \mathrm{ml}$ keine Soforttypreaktion und nach zwei Tagen eine fragliche Spättypreaktion (+) auf. Im Intrakutantest kam es ebenfalls zu keinen Soforttypreaktionen, jedoch nach zwei Tagen zu deutlichen Spättypreaktionen bei Konzentrationen von $0,08 \mathrm{mg} / \mathrm{ml}(++)$ und $0,8 \mathrm{mg} / \mathrm{ml}(+++)$. Im Lymphozytentransformationstest konnte mit Blutzellen des Patienten ein deutlich erhöhter Stimulationsindex im Vergleich zu zwei Kontrollen aufgezeigt werden.

Diese Ergebnisse weisen auf eine T-Zellvermittelte Kontaktallergie gegen Colistin als Ursache der Atemnot hin, erklärte Varga. Die Möglichkeiten einer Toleranzinduktion seien hier zu diskutieren, wie sie in der Literatur bereits beschrieben wurden.

Angelika Bauer-Delto

Varga R, Friedl T, Vogel S, Summer B, Thomas P, Ruëff F, Przybilla B. Atemnot durch Kontaktallergie auf Colistin?

\section{Molekulare Erforschung neuer Allergene}

Die molekulare Forschung konnte in den letzten Jahren wesentliche Fortschritte bei der Identifizierung von Allergenen erzielen, die beispielsweise für anaphylaktische Reaktionen auf bestimmte Nahrungsmittel maßgeblich oder für Kreuzreaktionen verantwortlich sind. So konnten Wissenschaftler vom Paul-Ehrlich-Insitut in Langen das Speicherprotein Gly $\mathrm{m} 5$ der Sojabohne als wesentlichen Marker für schwere anaphylaktische Reaktionen und als Majorallergen bei europäischen Kindern mit Sojaallergie identifizieren. Die Untereinheit Gly m 5.03 des Sojaspeicherproteins weist die höchste IgE-Bindungsfrequenz auf, berichtete Dr. Dirk Schiller. Seine Arbeitsgruppe untersucht derzeit bei einer Serie von Sojaallergikern, welche Bereiche auf der Proteinoberfläche von Gly $m$ 5.03 lgE-Bindungsstellen repräsentieren. Hierbei konnten bei einem Patienten mit Anaphylaxie gegen Soja, der nur auf Gly $\mathrm{m}$ 5.03 eine IgE-Reaktivität zeigte, die Aminosäuren 293-307 als mögliches IgE-Epitop nachgewiesen werden.
Auf der Suche nach Allergenen, die für die häufige Kreuzsensibilisierung gegen Hundeund Katzenhaare verantwortlich sein könnten, hat eine Arbeitsgruppe um Dr. Christiane Hilger vom Centre de Recherche Public de la Santé in Luxemburg das Majorallergen Can $\mathrm{f} 6$ dingfest gemacht. Das Protein konnte beim Hund in Speicheldrüsen und Hautschuppen nachgewiesen werden. ELISA-Screenings bei Seren von 44 Patienten mit einer Allergie gegen Hunde- und Katzenhaare ergaben bei $61 \%$ lgE-Antikörper gegen Can $\mathrm{f}$ 6. Die IgE-Reaktivität gegen rCan $\mathrm{f} 6$ wies eine hohe Korrelation mit einer IgEReaktivität gegen das Katzenallergen rFel d 4 auf. IgE-Kreuzreaktionen zwischen Can $\mathrm{f} 6$ und Fel $d 4$ konnten mittels Inhibitionsexperimenten nachgewiesen werden.

Auch in Insektengift konnten verschiedene neue Allergene detektiert werden. Forscher vom Institut für Biochemie und Molekularbiologie der Universität Hamburg wiesen in Zusammenarbeit mit der TU München die Relevanz und das allergene Potenzial meh- rerer selten vorkommender Proteine (low abundance proteins) nach. So konnten beispielsweise im Gift der Honigbiene sowie der Wespe IgE-reaktive Proteine von 200 kDa nachgewiesen werden, die der Familie der Vitellogenine angehören. Api m 12 und Ves $v 6$ weisen eine sequenzielle Identität von $40 \%$ auf und konnten als neues Paar kreuzreaktiver Panallergene identifiziert werden, berichtete Dr. Simon Blank.

Angelika Bauer-Delto

Schiller D, Becker S, Kühne Y, BallmerWeber B, Vieths S, Holzhauser T. Mutational epitope analysis of Gly m 5.03, a subunit of the major allergen in soybean beta-conglycinin

Hilger C, Swiontek K, Arumugam K, Lehners $C$, Hentges F. Identification of a new major dog allergen, Can $\mathrm{f} 6$, highly cross-reactive with Fel d 4

Blank S, Michel Y, Bantleon F, Seismann H, McIntyre M, Ollert M, Spillner E. Evaluation of the lgE sensitizing potential of novel hymenoptera venom allergens 